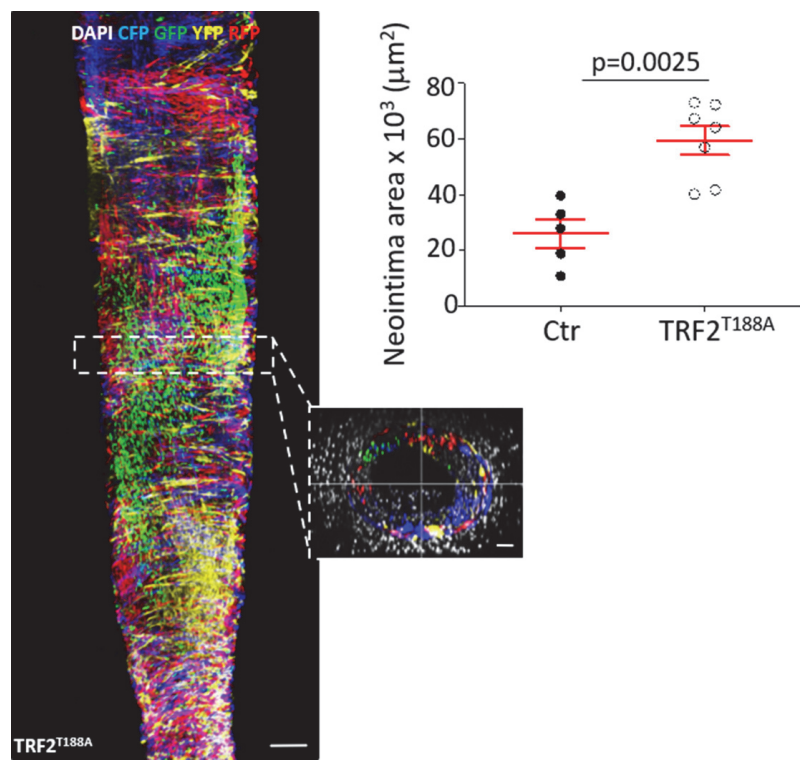

Abstract BS34 Figure 1

driving inflammation through immune cell recruitment in vascular disease. Targeting senescence and senescence-associated inflammation in particular, could limit injury-induced neointima formation and neoatherosclerosis.

Conflict of Interest No

\section{BS35 PONTIN REGULATES CARDIAC REMODELLING BY MODULATING THE HIPPO PATHWAY IN CARDIOMYOCYTES}

Bayu Lestari, Thuy Anh Bui, Sukhpal Prehar, Min Zi, Efta Triastuti, Ardiansah Bayu Nugroho, Ryan Potter, Nicholas Stafford, Elizabeth Cartwright, Alicia D'Souza, Delvac Oceandy. Division of Cardiovascular Sciences, FBMH, University of Manchester, Manchester, UK

\subsection{6/heartjnl-2021-BCS.233}

The development of heart failure (HF) is characterised by adverse remodelling events such as hypertrophy, fibrosis, and apoptosis, which together can alter the size, shape, and function of the heart. Identification of new factors that are involved in these processes is important in developing new strategies for HF therapy. Pontin (RUVBL1) is a member of the AAA+ protein family that regulates embryonic zebrafish heart development; however, its role in the adult mammalian heart is unknown. Our previous studies have found that Pontin can induce the activity of the major downstream effector of the Hippo pathway YAP, which is known to play an essential role in mediating cardiac remodelling. Thus, in this study, we aim to investigate the roles of Pontin in mediating adverse cardiac remodelling.

Pontin knock-down (KD) and overexpression (OE) in primary neonatal rat cardiomyocytes (NRCM) were achieved by siRNA-mediated gene silencing and adenoviralmediated overexpression, respectively. We found that Pontin KD negatively modulates NRCM proliferation, represented by a significant reduction in Ki67-positive cells and EdU-incorporation compared to control. Moreover, Pontin KD significantly reduces the level of active YAP, nuclear YAP localisation, and YAP transcriptional activity in NRCM. In contrast, Pontin OE induced NRCM proliferation by approximately 1.6 folds as determined by Ki67 staining and EdU incorporation assay. YAP activity and nuclear translocation were also significantly increased following Pontin OE. We also found that Pontin OE reduced cardiomyocyte hypertrophy and apoptosis (TUNEL staining) in response to Angiotensin II stimulation, whereas KD of Pontin increased Angiotensin-stimulated hypertrophy and apoptosis.

To study the role of Pontin in vivo, we generated a mouse model with an inducible cardiomyocyte-specific knockout of Pontin (PontinicKO) by crossing Pontinflox/flox mice with $\alpha \mathrm{MHC}-\mathrm{MerCreMer}$ transgenic mice. Induction of Pontin ablation was achieved by intraperitoneal injection with tamoxifen. We found that PontinicKO mice exhibited a severe cardiomyopathy phenotype at four weeks after tamoxifen injection, which was characterised by a significant reduction of ejection fraction, increased cardiac fibrosis and hypertrophy, and profound cardiomyocyte apoptosis.

In conclusion, our study has identified Pontin as a key regulator of cardiac remodelling, likely by modulating the Hippo pathway. Further studies are needed to explore the therapeutic potentials of Pontin in controlling cardiac remodelling.

Conflict of Interest None

\section{BS36 SELECTIVE ACTIVATION OF PRIMED VASCULAR SMOOTH MUSCLE CELLS}

Jordi Lambert, Sebnem Oc. Cambridge university, Cambridge, UK

10.1136/heartjnl-2021-BCS.234

Dysregulation of vascular smooth muscle cells (VSMCs) is a hallmark of vascular diseases including aneurysm and atherosclerosis. VSMCs in healthy vessels maintain a contractile and quiescent phenotype, but can be 'activated' and undergo phenotypic switching to a synthetic, proliferative state when under stress. Previously, we have shown that VSMC expansion after carotid ligation and in atherosclerotic lesions is oligoclonal, meaning activation is restricted to few VSMCs (Chappell et al., 2016, PMID: 27682618). Further to this, we identified a small population of Sca1+ VSMCs with reduced expression of contractile markers in healthy vasculature (Dobnikar et al., 2018, PMID: 30385745), which we hypothesize may have increased responsiveness. However, the mechanisms behind selective VSMC activation and the relevance of Sca1+ cells in disease are yet to be elucidated.

To investigate VSMC activation dynamics, we used a murine carotid ligation injury model, which acutely induces VSMC proliferation. We conducted single-cell RNA-sequencing (scRNA-seq) 5 days after injury, corresponding with the onset of proliferation. Dimensionality reduction showed that injury resulted in a gradient of VSMC phenotypes from contractile to proliferative, with no evidence of a distinct response by a dedicated progenitor population. To characterise this gradual phenotypic change, we performed trajectory analysis, where cells are ordered in 'pseudotime' based on gene expression similarity. Trajectory inference identified two injury response paths of which only one was associated with the transition to active cell proliferation, alongside extracellular matrix organization, and cell adhesion. The second path was enriched for genes associated with protein refolding. 